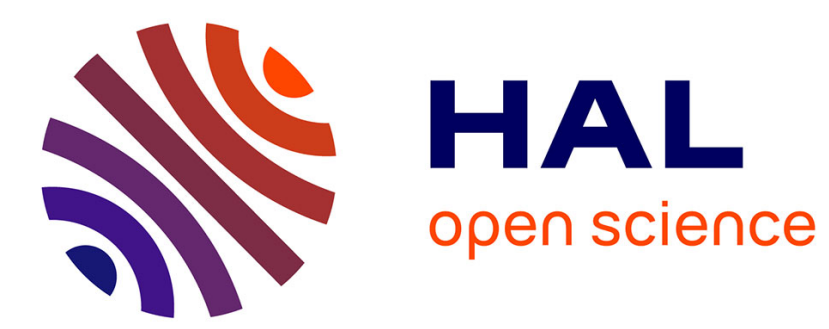

\title{
Quantum half-adder Boolean logic gate with a nano-graphene molecule and graphene nano-electrodes
}

\author{
Saurabh Srivastava, Hiori Kino, Christian Joachim
}

\section{To cite this version:}

Saurabh Srivastava, Hiori Kino, Christian Joachim. Quantum half-adder Boolean logic gate with a nano-graphene molecule and graphene nano-electrodes. Chemical Physics Letters, 2017, 667, pp.301306. 10.1016/j.cplett.2016.11.009 . hal-01712749

\section{HAL Id: hal-01712749 \\ https://hal.science/hal-01712749}

Submitted on 19 Jun 2018

HAL is a multi-disciplinary open access archive for the deposit and dissemination of scientific research documents, whether they are published or not. The documents may come from teaching and research institutions in France or abroad, or from public or private research centers.
L'archive ouverte pluridisciplinaire HAL, est destinée au dépôt et à la diffusion de documents scientifiques de niveau recherche, publiés ou non, émanant des établissements d'enseignement et de recherche français ou étrangers, des laboratoires publics ou privés. 


\title{
Quantum Half-Adder Boolean Logic Gate with a Nano-Graphene molecule and Graphene Nano-electrodes
}

\author{
Saurabh Srivastava ${ }^{\mathrm{a}, *}$, Hiori Kino ${ }^{\mathrm{a}}$, Christian Joachim ${ }^{\mathrm{b}, \mathrm{a}, *}$ \\ ${ }^{a}$ WPI-MANA, National Institute for Material Sciences, 1-1 Namiki, Tsukuba, Ibaraki, \\ Japan \\ ${ }^{b}$ CEMES-CNRS, 29 Rue J. Marvig, BP 4347, 31055 Toulouse Cedex, France
}

\begin{abstract}
A molecule Boolean 1/2-adder is designed and the XOR and AND truth table calculated at $+0.1 \mathrm{~V}$ using 4 graphene electrodes. It functions with level repulsion and destructive interferences effects using 4 molecule electronic states in a quantum Hamiltonian computing approach (QHC) with the abrupt change of the molecular orbital weight of those 4 calculating states as a function of the logical input configuration. The logical inputs enter rotating the two nitro groups of the central board. With QHC, a complex Boolean digital function can be implemented employing the same graphene material for interconnects and the molecule calculating parts.

Keywords: Graphene, Logic Gates, Quantum Transport, Quantum

Computing, Molecular electronics, Half-Adder
\end{abstract}

\section{Introduction}

The continuing miniaturization of electronic circuits triggers the ultimate search for single molecule based electronics where a molecule embedded between many electrodes will perform a digital Boolean function by itself[1, 2]. One pos-

5 sibility is to interconnect molecular switches [3, 4, rectifiers [5, 6] or amplifiers [7]

\footnotetext{
* Corresponding author

Email addresses: SRIVASTAVA.Saurabh@nims.go.jp (Saurabh Srivastava), christian.joachim@cemes.fr (Christian Joachim)
}

Preprint submitted to Chemical Physics Letters

November 2, 2016 
together using metallic nanowires 8 to construct the circuit. This approach is now progressively abandoned due to the absence of power gain per molecular device 9. Semi-classically designed intramolecular circuits 10, have also been explored [1] facing now the problem of the exponential decay of the output current with an increase of the spatial extension of the molecular circuit [12].

To solve those gain and exponential decay problems still with the objective to embark a calculating unit on a single molecule, a new quantum computing approach was proposed 13. When prepared in a non-stationary state, the quantum Hamiltonian computing (QHC) logic gate beneficiates from the spontaneous Heisenberg-Rabi quantum oscillations of the quantum system to run a quantum computation with classical inputs 14. By measuring the effecting Heisenberg-Rabi oscillations frequency using metallic nano-electrodes [15], different Boolean logic operations can be performed in parallel and within the same quantum system[16]. The functioning of a Boolean QHC gate is based on the quantum level repulsion effect together with the control of constructive and destructive quantum interferences affecting tunneling transport. Quantum design rules for QHC single molecule logic gates have been reported by Renaud et al.[13]. Following this approach, Soe et al. 17] have demonstrated experimentally a NOR logic gate function using a tri-naphthalene molecule whose frontiers molecular orbitals were manipulated by single $\mathrm{Au}$ atom contact to each of the naphthyl branch. Formally, A different QHC design was used to structure a single molecule NOR-AND 18 gate. For the previous hybrid 1/2-adder Boolean logic functions 19, 20, rotation of two lateral nitro chemical groups were also providing the classical digital inputs to the QHC gate here with a direct conversion of each rotation angle in quantum information. This conversion can be exactly measured by decomposing each molecular state on its Slater atomic basis set. It shows how a given $\mathrm{NO}_{2}$ rotation angle is encoded on the normalized atomic orbital coefficients of certain molecular states.

For these QHC molecule Boolean logic gates, metallic nano-electrodes were used to measure the logical outcome of the gates. Such metal-organic hybrids lead to a very low intensity tunneling current per output due to the large differ- 
ence in electronic structure between the central calculating molecule unit and its metallic measuring nano-electrodes. To design more complex QHC molecule logic gates (for exploring how the QHC approach can do better in term of decoherence as compared to the quantum qubit approach 21]) and for beneficiating in application of tunneling current intensities larger than a few nano-ampere, the calculating molecule and the measuring electrodes must be made of the same material. The graphene planar 2D material with its tunable electronic, mechanical and electronic properties [22, 23, 24] is an excellent candidate for a monolithic approach of QHC molecular logic gates.

In this letter, we demonstrate theoretically that a double nitro functionalized nano-graphene molecule (NGM) connected using graphene electrodes is performing a 1/2 adder Boolean operation in the $\mathrm{QHC}$ way using the rotation of the nitro-groups as the classical digital inputs. The first section is describing the QHC NGM design. Section 2 is recalling the technique used in this letter to calculate all the tunneling current intensity passing through the central NGM. Section 3 is describing the functioning of our $1 / 2$ adder QHC NGM. In conclusion, we compare our design with a classical $1 / 2$ adder electronic circuit.

\section{The Boolean $1 / 2$ adder $\mathrm{QHC}$ molecular design}

On Fig. 1] a central NGM is chemically bonded to 4 ultra-short 3 phenyls in length and 2 in width graphene nano-ribbon (GNR1 to GNR4). The core of the NGM molecule was designed starting from a coronene molecule extended asymmetrically by phenyl rings to be able to distinguish an AND and an XOR functionality by its MO weight at the measuring point. Four different conjugated boards were tested not to extend too much this central NGM. Each GNR is connected to independent semi-infinite graphene electrode whose electronic band structure is well defined using periodic boundary conditions 30 . The central NGM is $2 \mathrm{D}$ crystal oriented in the same direction as the graphene electrodes to beneficiate from a complete homo electronic structure between the NGM and 
synthesized 25]. The Fig. 1 NGM circuit may be nanoscale fabricated for its interconnection parts using a high precision $\mathrm{He}^{+}$scattering [26] and using scanning tunneling microscope (STM) single atom vertical manipulations for the atomic scale part as already demonstrated at the surface of $2 \mathrm{D} \mathrm{MoS}_{2}$ materials 27. In

70 Fig. 1 the A and B nitro group inputs were chemically bonded to the NGM for distributing the logical inputs between the XOR and AND part of the QHC circuit without requiring ancillary internal or external molecular wires as commonly performed in standard electronic Boolean logic circuits. In electronics, the output of a given gate along the circuit is generally required to distribute its output signal at many locations of the circuit at the same time. Our present molecule QHC design can be considered as a practical implementation of the formal $\mathrm{QHC}$ 1/2-adder [16] where it was shown how to position the logical inputs along the QHC quantum graph to avoid this cascading problem. Two fluorine atoms were also added to the NGM to optimize, as presented in Fig. 44 the distribution of the frontier molecular orbitals over the NGM depending on the logical input configuration as discussed later. With respect to the graphene electrode plane, a planar nitro is encoding for a "0" binary logical input and a perpendicular one for a "1" logical input. This encoding was chosen because of the non-zero switching energy certainly required to input a logical "1" on the NGM by STM rotating a single $\mathrm{NO}_{2}$ group, as it was already demonstrated for a single ter-butyl group [28, the native nitro configuration being planar 29]. A nitro group is a small but very efficient electron acceptor group, very active in interacting with a conjugated molecule while co-planar with it [30, 31].

\section{The Calculation Technique}

Through the Fig. 1 QHC circuit and using Landauer formula 32, 33, the current intensity $I$ was calculated between any two graphene electrodes and when applying a bias voltage $\mathrm{V}$ using :

$$
I=\frac{e}{\pi \hbar} \int_{E_{f}}^{E_{f}+e V} T(E) d E,
$$


where $E_{f}$ is the Fermi energy of a given graphene electrode and $T(E)=$ Trace $\left(t(E) t^{\dagger}(E)\right)$, the electronic transparency between those two graphene electrodes. $t(E)$ is the top left block of the multi-channel elastic scattering matrix describing the scattering process through the central NGM. The complete multichannel scattering matrix was calculated exactly using the elastic scattering quantum chemistry (ESQC) method[16, 34, 35]. A full valence semi-empirical Hamiltonian was used in ESQC to describe the central molecule along with its 4 short GNR molecular wires and the graphene electrodes. This Hamiltonian was constructed with the full valence extended Hückel molecular orbitals (EHMO) basis set using Hoffmann parameters [36, 37] for all the C, N, O, F, and $\mathrm{H}$ atoms. A discussion about the Hoffmann parameters preferred over Cérda parameters [38, 39] can be found in [30] for graphene. Using those EHMO parameters, the calculated graphene electrode band structure shows a relative energy position of the Dirac cone crossing point at $E=E_{f}=-10.53 \mathrm{eV}$.

\section{The functioning of the nano-graphene QHC $1 / 2$ adder}

In Fig. 2, the calculated $\mathrm{T}(\mathrm{E})$ spectra for the XOR and AND outputs are presented as a function of the A and B logical input configurations. The cross "leakage" T(E) spectra between one XOR and one AND graphene electrodes, respectively, through the NGM were also calculated and according to equation 1 lead to a less than $0.01 \mu \mathrm{A}$ tunneling current intensity for a $0.1 \mathrm{~V}$ bias voltage as compared with the direct XOR and AND outputs. This is the maximum calculated leakage current from one XOR to one AND graphene electrodes and for only the $(1,1)$ input configuration. A $0.01 \mu \mathrm{A}$ current intensity on the AND for $(1,1)$ is not very noticeable as compared to the $2 \mu \mathrm{A}$ measured output current for this input configuration. All the others cross leakage current are one order of magnitude lower than $0.01 \mu \mathrm{A}$ at $0.1 \mathrm{~V}$ for all the other logical input configurations. The $T(E)$ spectra are showing up some $\pi$ and $\pi^{*}$ resonances together with destructive interference patterns i.e. very pronounced s in the $\mathrm{T}(\mathrm{E})$ spectrum for example at $E=-10.5 \mathrm{eV}$ for the $\mathrm{XOR}(0,0)$ and at $E=-10.35$ 
$\mathrm{eV}$ for the AND $(0,1) /(1,0)$ configurations. The functioning of the Fig. 1 $\mathrm{QHC}$ $1 / 2$ adder is based on the quantum level repulsion effect and on an optimized energy positioning of those tunneling destructive interferences relative to the graphene Fermi energy. As also demonstrated recently with a formal Boolean Hamiltonian, having two outputs for the same QHC system and measured in the same energy range requires that the weight of the molecular orbitals at measurement points (here $\mathrm{C} 1$ and $\mathrm{C} 2$ ) be quite exclusive as a function of the input configuration[16].

Let us first start with the level repulsion effect. Among all the $\pi$ and $\pi^{*}$ molecular orbitals of the central NGM and in an energy range $2 \mathrm{eV}$ around the Fermi energy, only a few are effectively contributing to the $1 / 2$ adder functioning because many have no molecular weight at the $\mathrm{C} 1$ and $\mathrm{C} 2$ chemical bonding sites of the graphene electrodes. We have identified on Fig. 2, the 4 main "calculating" molecular states of the central NGM i.e. the T(E) spectra resonances contributing the most to the $1 / 2$ adder functioning. They have been labeled 1 to 4 for mono-electronic quantum states $|1\rangle$ to $|4\rangle$ (For completion and in the Suppl. Mat. 5. they are also labeled on the corresponding complete spectrum of this central NGM alone). For clarity, Fig. 3 is only showing up the energy position of those 4 states in a quantum level correlation diagram. There is a clear level repulsion effect in action on state $|1\rangle$ shifted up in energy by about 0.3 $\mathrm{eV}$ and on state $|3\rangle$ by about $0.2 \mathrm{eV}$ by going from a parallel to a perpendicular nitro conformations for AND and XOR respectively.

Based on this level repulsion effect, the functioning of the Fig. 1 1/2 adder QHC gate is also relaying on the control by the nitro angles of the $T(E)$ constructive and destructive interference patterns. Notice that, those patterns also depend on the chemical structure of contact $\mathrm{C} 1$ and $\mathrm{C} 2$. For a $(0,0)$ logical input i.e. for two planar nitro's, there is a very pronounced $T(E)$ destructive interference between states $|1\rangle$ and $|4\rangle$ at $E=-10.49 \mathrm{eV}$ for the XOR $T(E)$ and 150 at $E=-10.51 \mathrm{eV}$ for the AND $T(E)$. Located near the Fermi energy and at low positive bias voltage, this will leads to a very low output current according to equation 1, ensuring the required zero logical output for both the XOR and 
the AND. Notice that, the two very sharp resonances located just below the Fermi energy are coming from states $|1\rangle$ and $|3\rangle$ which are only weakly coupled to the graphene electrodes for $(0,0)$. They will not contribute to the XOR and AND logical outputs in this case.

The $(0,0)$ input destructive interference patterns are drastically changed by rotation of the nitro group one after the other from a planar to a perpendicular conformation (See Suppl. Mat. 6 for the detail T(E) interference pattern changes following the progressive rotation of the two nitro groups). For the $(0,1)$ and $(1,0)$ input configurations, the $(0,0)$ XOR central destructive interference observed previously at $E=-10.49 \mathrm{eV}$ is now shifted below the Fermi energy and state $|3\rangle$ leads now to a very sharp constructive resonance positioned up the Fermi energy at $E=-10.50 \mathrm{eV}$. This results from the restoration of the electronic coupling between state $|3\rangle$ and the two GNR3 and GNR4 nano-contacts after reaching a perpendicular conformation for only one nitro group having the effect of reintroducing an electronic density expansion of $|3\rangle$ at contact $\mathrm{C} 2$ (see also below Fig. 4). The resonance from state $|2\rangle$ is still very sharp and will not contribute to the tunneling current because it is deeply attenuated by the central destructive interference located at $E=-10.55 \mathrm{eV}$. Also for $(0,1)$ and $(1,0)$, the AND central destructive interference is shifted up and above the Fermi energy at $E=-10.35 \mathrm{eV}$. Here, state $|3\rangle$ is still leading to a very sharp resonance below the Fermi energy. State $|3\rangle$ will not contribute to the AND tunneling current output.

For the $(1,1)$ input configuration, the XOR central destructive interference is still located below the Fermi energy and state $|2\rangle$ resonance remains below the Fermi energy. More important, the resonance coming from $|3\rangle$ is still very sharp and now shifted up further away from the Fermi energy. This means that at low bias voltage and according to equation 1 it will not contribute to the XOR tunneling current. Also for $(1,1)$, the AND destructive interference between $|1\rangle$ and $|4\rangle$ which was located for $(0,1)$ and $(1,0)$ at $E=-13.35 \mathrm{eV}$ is now no more existing. The state $|1\rangle$ resonance has now reached $E=-10.49 \mathrm{eV}$ with a large width resonating Lorentzian shape. At low positive voltage and according to 
equation 1, this will lead to the AND output functioning.

According to the $\mathrm{T}(\mathrm{E})$ spectra in Fig. 2, both the XOR and AND output status must be measured in an energy range about $0.1 \mathrm{eV}$ up the Fermi energy. As a consequence, the chemical structure of the $\mathrm{C} 1$ and $\mathrm{C} 2$ contacts have been designed differently to be able to distinguish between the XOR and AND output because they are resonating almost at the same energy. The effect of this different design between the $\mathrm{C} 1$ and $\mathrm{C} 2$ contacts can be observed around the state $|4\rangle$ resonances by comparing the structure of the corresponding resonance massif for the XOR and the AND. It was demonstrated in Hosoki et al. 27] that when measuring at the same energy the 2 different logical outputs on the same QHC system (here the XOR and the AND), one need a very abrupt change of the weight of the corresponding orbitals as a function of the logical input to be able to spatially distinguish 2 outputs at the same energy. As presented in Fig. 4, this is exactly what was achieved with the Fig. 1 design thanks also to the substitution of 2 hydrogen atoms by 2 fluorine atoms on the central NGM. By representing states $|1\rangle$ and $|3\rangle$ corresponding molecular orbital normalized weight distribution on the NGM, it is clear that only the input configuration $(1,1)$ is leading to a large orbital weight for $|1\rangle$ at C1 for the AND. Only input configurations $(0,1)$ and $(1,0)$ are doing the same for $|3\rangle$ at $\mathrm{C} 2$. This exclusive distribution of the molecular orbital spatial weight determines the good functioning of our $1 / 2$ adder QHC gate. It is exactly the chemical implantation of the formal Boolean Hamiltonian studied in Dridi et. al [16].

\section{Discussion}

Using the Fig. $2 \mathrm{~T}(\mathrm{E})$ spectra in equation 1 to calculate the $0.1 \mathrm{~V}$ low voltage tunneling current intensity passing through the central NGM at C1 and C2, the 1/2 adder Boolean truth table is presented in Table1. According to the standard C. Shannon like design[40, a Boolean 1/2 adder is normally requiring the interconnection of a minimum of 4 transistors and a dedicated distribution of the voltage logical inputs among those transistors using a few wires and 
circuit nodes. The presented 232 atoms nano-graphene calculating molecule has no transistors nor apparent internal molecular wires like circuitry. Among the $168 \pi$ and $\pi *$ quantum states of the Fig. 1 central nano-graphene molecule, mainly 4 states are "calculating" as presented in Fig. 3. The NGM quantum states $|1\rangle$ and $|3\rangle$ are shifted up because of the rotation of 2 strong electron acceptor groups. Those are classical mechanical inputs which cancertainly be activated by scanning probe techniques but has not been experimented yet. State $|4\rangle$ is very important to create the $(0,0)$ central destructive interference and state $|2\rangle$ to moderate the $(1,1)$ XOR output current to ensure a low output current for this input configuration. Nearby in energy to those 4 states, a few more are moderating the level repulsion acting on $|1\rangle$ and $|3\rangle$ to limit, for example, the shifting up of the resonances $|1\rangle$ and $|3\rangle$ while rotating the nitro 225 groups. All the other NGM quantum states can be considered as structural states ensuring the chemical stability of the central nano-graphene molecule. Importantly the XOR and AND output current intensities are quite large. First because the tunneling electrons have not to be transferred through the complete NGM molecular structure to deliver each output status. Second because the measuring nano-pads are homo electronic with the central calculating nanographene, a way to optimize the contact conductance at $\mathrm{C}_{1}$ and $\mathrm{C}_{2}$.

Our nano-graphene QHC 1/2-adder demonstrates how a small molecular nanostructure can performed a rather complex logic function: a binary addition with a carry without using an extended molecular system but more important without requiring any power gain inside the calculating board. The nonnecessity of a power gain comes from the fact that the output status of the gate are measured without passing a current through to the entire molecule. Contrary to previous proposed QHC 1/2- adder design 20, it results from a local measurement. This new $\mathrm{QHC}$ design opens the search for more complex and monolithic QHC intramolecular logic gates based on intramolecular quantum information exchanges where the multiple output status are measured locally. 


\section{Acknowledgement}

SS thanks the MANA-NIMS Theory-Experiment Fusion Research Program and the NIMS computation facilities. CJ thanks the WPI MANA program for

financial support and the constant encouragements of Pr. M. Aono during this work.

\section{References}

[1] C. Joachim, J. K. Gimzewski, A. Aviram, Electronics using hybridmolecular and mono-molecular devices, Nature 408 (2000) 541-548.

[2] W. X. Zhang, Voltage-driven spintronic logic gates in graphene nanoribbons, Scientific Reports 4 (2014) 6320-1-6320-4.

[3] C. P. Collier, E. W. Wong, M. Belohradsk, F. M. Raymo, J. F. Stoddart, P. J. Kuekes, R. S. Williams, J. R. Heath, Electronically configurable molecular-based logic gates, Science 285 (1999) 391-394.

[4] C. P. Collier, G. Mattersteig, E. W. Wong, Y. Luo, K. Beverly, J. Sampaio, F. M. Raymo, J. F. Stoddart, J. R. Heath, A [2]catenane-based solid state electronically reconfigurable switch, Science 289 (2000) 1172-1175.

[5] R. M. Metzger, All about (n-hexadecylquinolin-4-ium-1yl)methylidenetricyanoquinodimethanide, a unimolecular rectifier of electrical current, J. Mater. Chem. 10 (2000) 55-62.

[6] R. M. Metzger, Electrical rectification by a molecule:the advent of unimolecular electronic devices, Acc. Chem. Res. 32 (1999) 950-957.

[7] C. Joachim, J. Gimzewski, An electromechanical amplifier using a single molecule, Chem. Phys. Lett. 265 (1997) 353-357.

[8] S. Ami, C. Joachim, Logic gates and memory cellsbased on single $c_{60}$ electromechanical transistors, Nanotechnology 12 (2001) 44-52. 
[9] R. Staddler, S. Ami, M. Forshow, C. Joachim, A memory/adder model based on single $c_{60}$ molecular transistors, Nanotechnology 12 (2001) 350357.

270 [10] J. Ellenbogen, J. Love, Architectures for molecular electronic computers. i. logic structures and an adder designed from molecular electronic diodes, Proc. IEEE 88 (2000) 386-426.

[11] N. Jlidat, M. Hliwa, C. Joachim, A semi-classical xor logic gate integrated in a single molecule, Chem. Phys. Lett. 451 (2008) 270-275.

[12] L. Lafferentz, F. Ample, H. Yu, S. Hercht, C. Joachim, L. Grill, Conductance of a single conjugated polymer as a continuous function of its length, Science 323 (2009) 1193.

[13] N. Renaud, M. Hliwa, C. Joachim, Quantum design rules for single molecule logic gates, Phys. Chem. Chem. Phys. 13 (2011) 14404-14416.

[14] N. Renaud, C. Joachim, Design and stability of nor and nand logic gates constructed with three quantum state, Phys. Rev. A 78 (2008) 062316-1062316-10.

[15] O. Faizy, G. Dridi, C. Joachim, Parallel quantum circuit in a tunnel junction, Sci. Rep. 6 (2016) 30198-1-30198-10.

[16] G. Dridi, R. Julien, M. Hliwa, C. Joachim, The mathematics of a quantum hamiltonian computing half adder boolean logic gate, Nanotechnology 26 (2015) 344003.

[17] W.-H. Soe, C. Manzano, N. Renaud, P. de Mendoza, A. D. Sarkar, F. Ample, M. Hliwa, A. M. Echavarren, N. Chandrasekhar, C. Joachim, Manipulating molecular quantum states with classical metal atom inputs: Demonstration of a single molecule nor logic gate, ACS Nano 5 (2011) 1436-1440.

[18] N. Renaud, M. Ito, W. Shangguan, M. Saeys, M. Hliwa, C. Joachim, A norand quantum running gate molecule, Chem. Phys. Lett. 472 (2009) 7479. 
[27] S. Hosoki, S. Hosaka, T. Hasegawa, Surface modification of $\operatorname{mos}_{2}$ using an stm, Appl. Surf. Sci. 60 (1992) 643-647.

[28] C. Loppacher, M. Barmmerlin, M. Guggisberg, O. Pfeiffer, E. Meyer, R. Luthi, R. Schlitler, J. K. Gimzewski, H. Tang, C. Joachim, Direct deter-

[19] I. Duchemin, C. Joachim, A quantum digital half adder inside a single molecule, Chem. Phys. Lett. 406 (2005) 167-172.

[20] I. Duchemin, N. Renaud, C. Joachim, An intramolecular digital 1/2-adder with tunneling current drive and read-outs, Chem. Phys. Lett. 452 (2008) 269-274.

[21] M. Nielsen, I. L. Chuang, Quantum Computation and Quantum Information, Cambridge University Press, London, 2000.

[22] A. K. Geim, K. S. Novoselov, The rise of graphene, Nat. Mater. 6 (2007) $183-191$.

[23] K. S. Novoselov, A. K. Geim, S. V. Morozov, D. Jiang, Y. Zhang, S. V. Dubonos, I. V. Grigorieva, A. A. Firsov, Electric field effect in atomically thin carbon films, Science 306 (2004) 666-669.

[24] K. S. Novoselov, A. K. Geim, S. V. Morozov, D. Jiang, M. I. Katsnelson, I. V. Grigorieva, S. V. Dubonos, A. A. Firsov, Two-dimensional gas of massless dirac fermions in graphene, Nature 438 (2005) 197-200.

[25] R. Dorel, C. Manzano, M. Grisolia, W. Soe, C. Joachim, A. M. Echavaren, Tetrabenzocircumpyrene: a nanographene fragment with an embedded peripentacene core, Chem. Comm. 51 (2015) 6932-6935.

[26] S. Nakaharai, T. Iijima, S. Ogawa, S. Suzuki, S.-L. Li, K. Tsukagoshi, S. Sato, N. Yokoyama, Conduction tuning of graphene based on defectinduced localization, ACS Nano 7 (2013) 5694-5700.

mination of the energy required to operate a single molecule switch, Phys. Rev. Lett. 90 (2003) 066107-1-066107-4. 
[29] A. Domenicano, G. Schultz, I. Hargittai, M. Colapietro, G. Portalone, P. George, C. W. Bock, Molecular structure of nitrobenzene in the planar and orthogonal conformations, Struct. Chem. 1 (1990) 107-122.

[30] S. Srivastava, H. Kino, C. Joachim, Contact conductance of a graphene nanoribbons with its graphene nano-electrodes, Nanoscale 8 (2016) 92659271.

[31] C. Patoux, C. Coudret, J. Launay, C. Joachim, A. Gourdon, Topological effects on intramolecular electron transfer via quantum interference, Inorganic Chemistry 36 (1997) 5037-5049.

[32] M. Buttiker, Y. Imry, R. Landauer, S. Pinhas, Generalized many-channel conductance formula with application to small rings, Phys. Rev. B 31 (1985) $6207-6215$.

[33] A. D. Stone, A. Szafer, What is measured when you measure a resistance?the landauer formula revisited, IBM J. Res. Dev. 32 (1988) 384-413.

[34] P. Doumergue, L. Pizzagalli, C. Joachim, A. Altibelli, A. Baratoff, Conductance of a finite missing hydrogen atomic line on si(001)-(2x1)-h, Phys. Rev. B 59 (1999) 15910-15916.

[35] K. S. Yong, D. M. Otalvaro, I. Duchemin, M. Saeys, C. Joachim, Calculation of the conductance of a finite atomic line of sulfur vacancies created on a molybdenum disulfide surface, Phys. Rev. B 77 (2008) 205429-1-2054299.

[36] R. Hoffmann, An extended hückel theory. i. hydrocarbons, J. Chem. Phys. 39 (1963) 1397-1412.

[37] M. Wolfsberg, L. J. Helmholz, The spectra and electronic structure of the tetrahedral ions $\mathrm{mno}_{4}^{-}, \mathrm{Cro}_{4}^{--}$, and $\mathrm{clo}_{4}^{-}$, J. Chem. Phys. 20 (1952) 837-843.

[38] J. Cerdá, F. Soria, Accurate and transferable extended hückel-type tightbinding parameters, Phys. Rev. B 61 (2000) 7965-7971. 
[39] D. Kienle, J. Cerdá, A. W. Ghosh, Extended hückel theory for band structure, chemistry, and transport. i. carbon nanotubes, J. of Appl. Phys. 100 (2006) 043714-1-043714-9.

[40] F. Carter, The molecular device computer: Point of departure for large scale cellular automata, Physica D 10 (1984) 175-194. 


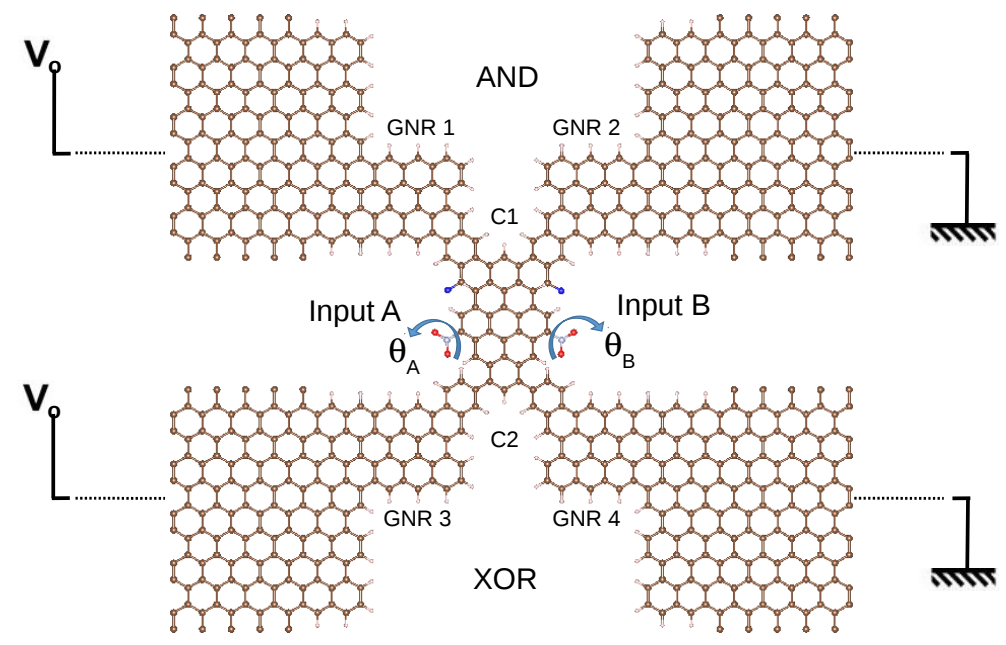

Figure 1: The atomic structure of the designed QHC 1/2 adder nano-graphene molecular logic gate with its 4 ultra-short GNR interconnecting molecular wires (GNR1 to GNR4) and its 4 graphene electrodes. The AND is measured at contact $\mathrm{C} 1$ and the XOR at contact $\mathrm{C} 2$. Contacts $\mathrm{C} 1$ and $\mathrm{C} 2$ are made of 2 phenyl rings each for the tunneling current to be measured between GNR1 and GNR2 for C1 and between GNR3 and GNR4 for C2. The graphene electrodes are semi-infinite to work with the graphene electronic band structurereproduced here using lateral cyclic boundary conditions. The two fluorine atoms are shown in blue whereas the two rotating nitro groups are shown in red. The presented geometry is for the nitro-groups in a planar configuration encoding for a $(0,0)$ logical input. 

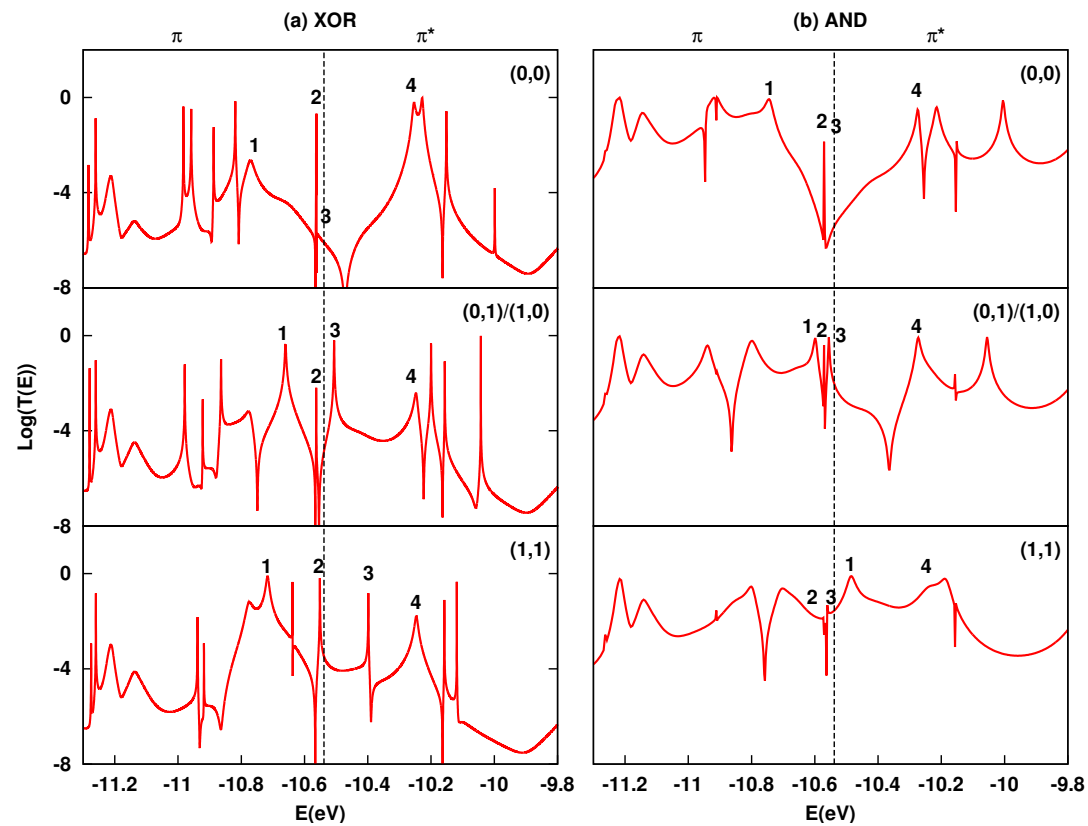

Figure 2: The ESQC calculated $T(E)$ electronic transmission coefficients for (a) the XOR and (b) the AND logical outputs as a function of the logical input configurations. For the $[-11.3 \mathrm{eV},-11.0 \mathrm{eV}],[-10.0,-9.8 \mathrm{eV}]$ XOR energy intervals and for the [-11.3 eV, $-10.9 \mathrm{eV}]$, $[-10.1 \mathrm{eV},-9.8 \mathrm{eV}]$ AND energy intervals, the T(E) remain unchanged while the resonances are moving in energy around the graphene Fermi energy. A zoom-in of this last part is also presented in Suppl. Mat. Fig. 6 For $(0,0)$, the destructive interference between $|1\rangle$ and $|4\rangle$ is ensuring a zero logical output current intensity. The level repulsion effect controlled by the nitro rotations is pushing up $|1\rangle$ and $|3\rangle$ for respectively the AND and the XOR. This is changing the $T(E)$ interference patterns leading to the adder functioning. States $|2\rangle$ for the XOR and $|2\rangle,|3\rangle$ for the AND are controlling the repulsion effect without playing a large role in the output current intensities. 


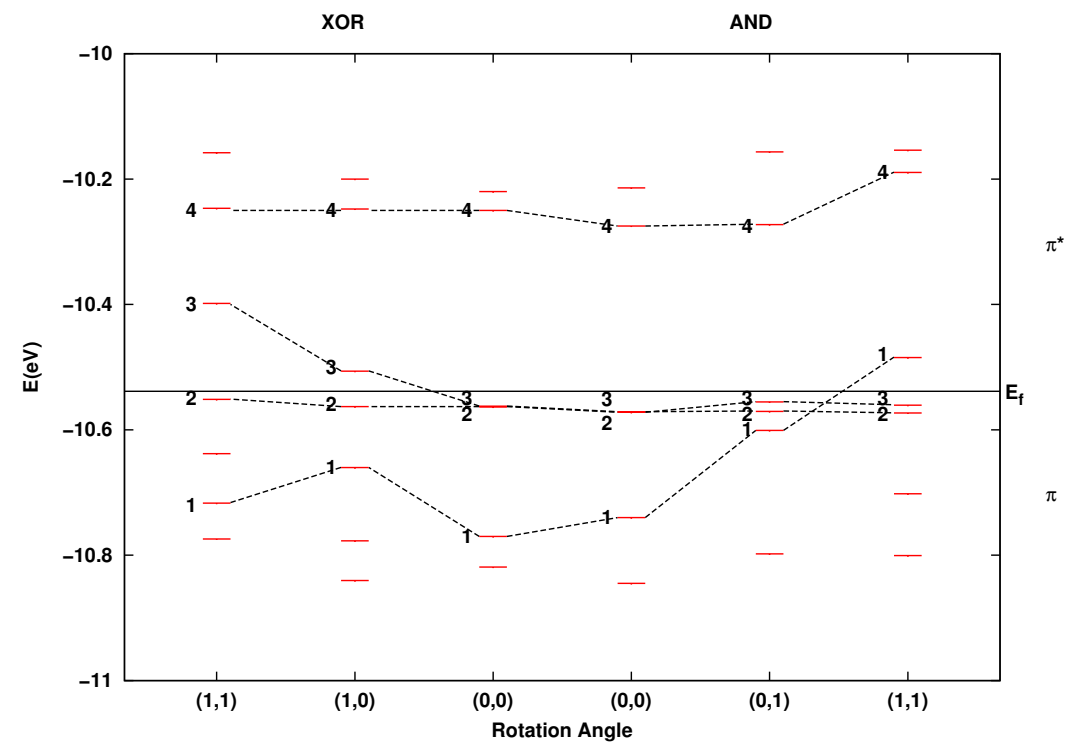

Figure 3: The energy level correlation diagram as a function of the logical input configuration for the XOR (left) and the AND (right) starting from the $(0,0)$ input (center). From a spectroscopy point of view, the nitro rotations are controlling the repulsion of $|3\rangle$ for the XOR and $|1\rangle$ for the AND. While interacting with the graphene electrodes through the ultrashort GNR molecular wires and as presented in Fig. 2, the destructive interference between $|1\rangle$ and $|4\rangle$ will complete the Boolean calculating behavior of the central nano-graphene molecule. The $\pi *|4\rangle$ is not repelled during the nitro rotation while $|1\rangle$ and $|3\rangle$ are moving up in energy towards $|4\rangle$. This energy level correlation was extracted from the complete mono-electronic energy spectrum around the graphene Fermi Level presented in Suppl. Mat. 5 Notice for $(0,0)$ the spectra difference between the XOR and the AND coming from the optimization of the molecular orbital distribution at the XOR and AND C2 and C1 contact points. 


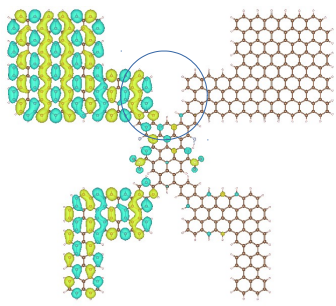

(a)

$(0,0)$

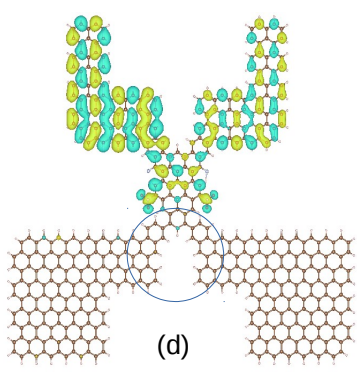

(d)

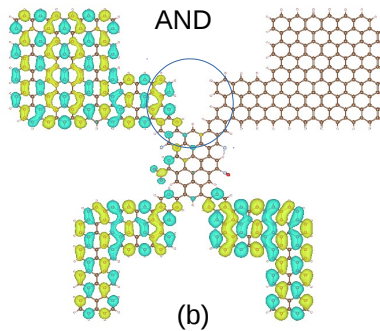

$(0,1) /(1,0)$

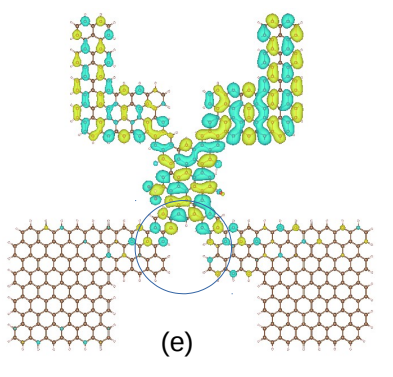

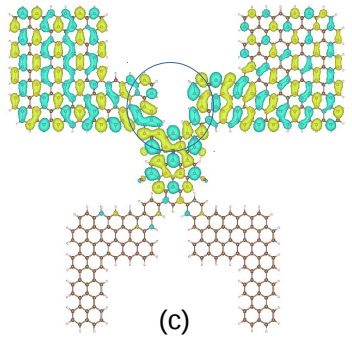

$(1,1)$

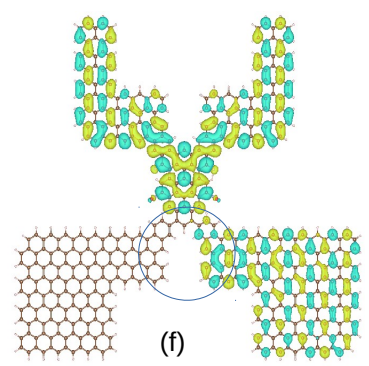

XOR

Figure 4: The change of the molecular orbital (MO) distribution of $|1\rangle$ for the AND and $|3\rangle$ for the XOR as a function of the nitro rotational input configurations. The central nano-graphene molecule and its $\mathrm{C} 1$ and $\mathrm{C} 2$ contact structure were designed in such a way that there is a maximum MO spatial weight at $\mathrm{C} 1$ for the $\mathrm{AND}$ and a maximum $\mathrm{MO}$ spatial weight at $\mathrm{C} 2$ for the XOR. For the AND output, rotating only one of the two nitro groups from a perpendicular to a planar configuration rapidly depopulates the $|1\rangle \mathrm{MO}$ at $\mathrm{C} 1$. The same at $\mathrm{C} 2$ for the XOR going from $(0,1),(1,0)$ to $(0,0)$ or $(1,1)$. This MO manipulation permits to measure the XOR and AND logical output status for exactly the same bias voltage. Blue circles are indicating the $\mathrm{C} 1$ and $\mathrm{C} 2$ contact position to appreciate the MO manipulation. Accordingly, the AND logical output is better defined than the XOR one for this current design (see also Table 1). 


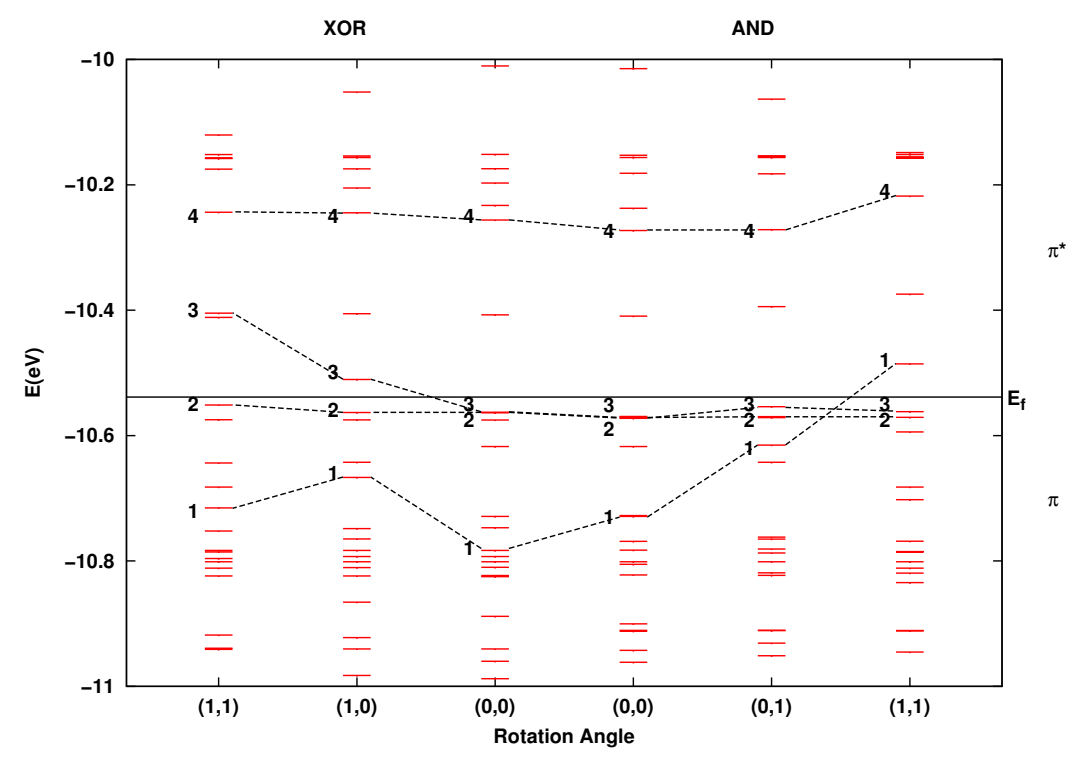

Figure 5: *

Supplementary Figure 1: The complete mono-electronic energy spectrum around the graphene Fermi Level in the relative $[-11.0 \mathrm{eV}:-10.0 \mathrm{eV}]$ energy range as a function of the logical input configurations. The Fig. 3 extracted "calculating" $\pi$ and $\pi *$ energy levels are also indicated with the same numbering than in Fig. 3 Many $\pi$ and $\pi *$ are not directly participating to the $1 / 2$ adder logic gate functioning because they are not electronic coupled to $\mathrm{C} 1$ and $\mathrm{C} 2$. They are indirectly contributing by moderating the energy level position shift of $|1\rangle$ to $|4\rangle$ during the nitro controlled level repulsion effects. 

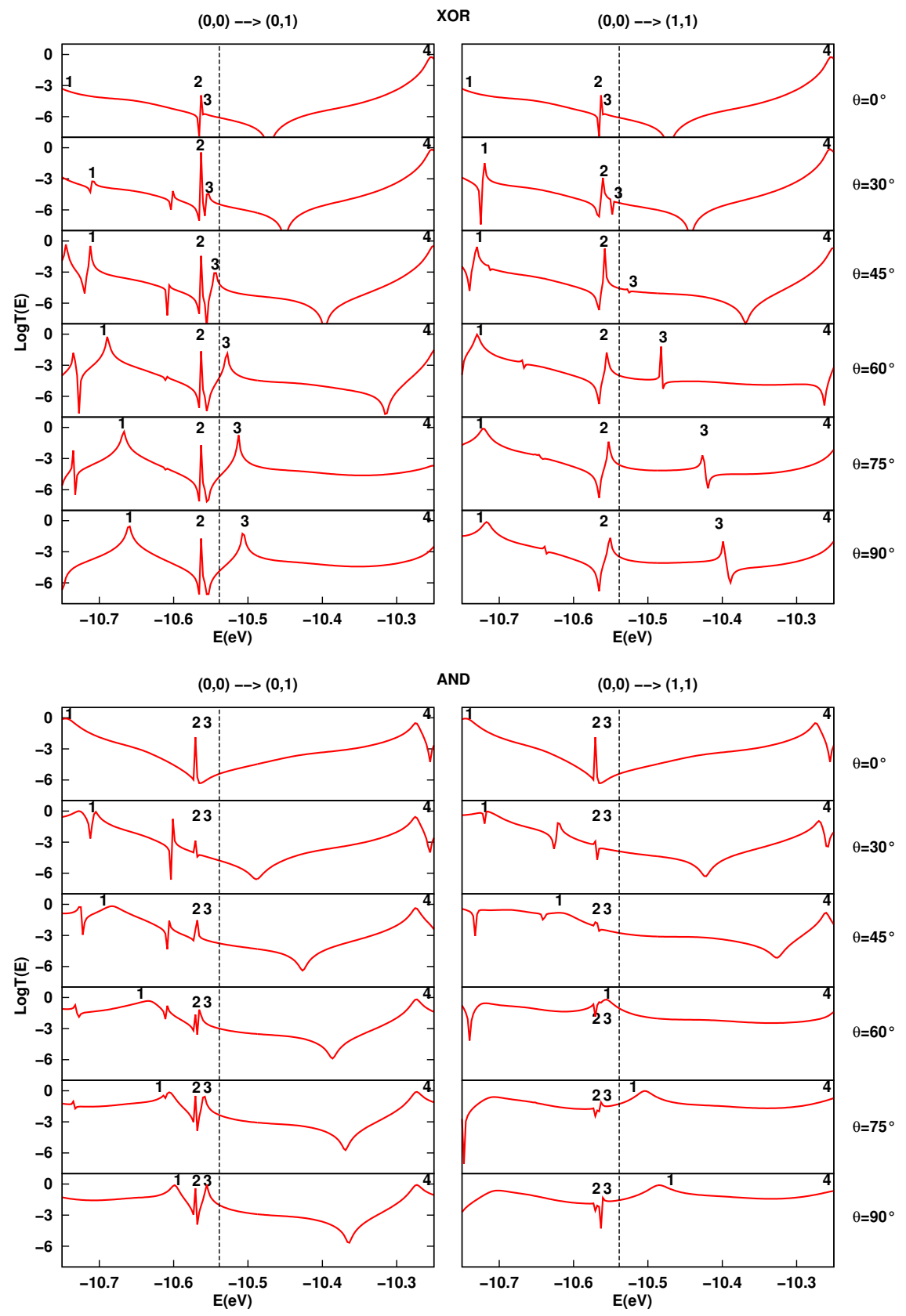

Figure 6: *

Supplementary Figure 2: The progressive transformation of the $T(E)$ spectra as a function of the nitro rotation angles $0^{\circ}, 30^{\circ}, 45^{\circ}, 60^{\circ}, 75^{\circ}$ and $90^{\circ}$ degrees in a small energy range around the graphene Fermi energy corresponding according to Fig. 2 only to the energy position of the "calculating" states $|1\rangle$ to $|4\rangle$. (a) is for XOR and (b) for AND logical output. This small energy range helps to appreciate how $0.1 \mathrm{eV}$ up the Fermi energy, equation 1 is leading to the output tunneling current intensities. 


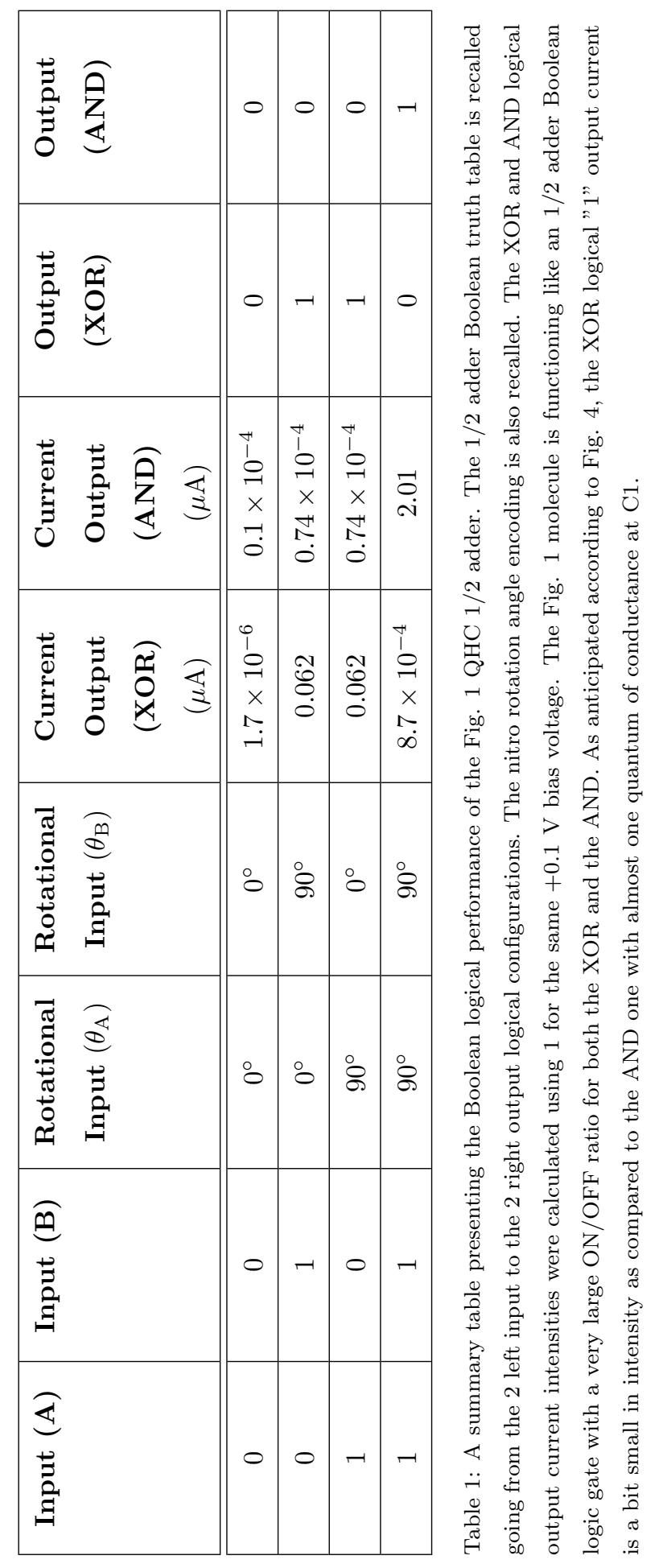

